\title{
Internet Distance Prediction Using Node-Pair Geography
}

\author{
Ankur Jain and Joseph Pasquale \\ Department of Computer Science and Engineering \\ University of California, San Diego \\ La Jolla, USA \\ ajain@cs.ucsd.edu,pasquale@cs.ucsd.edu
}

\begin{abstract}
Predictive methods for learning network distances are often more desirable than direct performance measurements between end hosts. Yet, predicting network distances remains an open and difficult problem, as the results from a number of comparative and analytical studies have shown. From an application requirements perspective, there is significant room for improvement in achieving prediction accuracies at a satisfactory level. In this paper, we develop and analyze a new, machine learning-based approach to distance prediction that seeks to capture and generalize geographical characteristics between Internet node pairs, instead of relying on direct and ongoing measurements of partial paths. We apply a basic algorithm in machine learning to demonstrate this idea and highlight the potential benefits that this method may offer over other popular methods that exist today.
\end{abstract}

Keywords- network latency; distance prediction; node-pairs; network geography

\section{INTRODUCTION}

Global-scale, distributed, and peer-to-peer applications and services have become highly pervasive in the modern Internet and continue to rapidly grow. In striving to achieve various performance goals, such as efficiency in content delivery, reliability, and durability, these systems must be able to intelligently select from a number of different communication paths and do so in a scalable manner. This requirement creates an intrinsic reliance of these services on knowledge of the relative proximities of hosts for large sets of potential targets. However, as this information is almost always not readily available, it must be obtained through direct measurements or, otherwise, be learned by some different means.

A popular idea and area of focus that aims to address this demand is Internet distance prediction (IDP). IDP refers to the problem of estimating delay-space proximities of Internet hosts without the use of explicit, on-demand performance measurements of the network paths connecting them. While the simple and easy option for nodes is to initiate communication to potential targets and acquire the necessary network distances, some important considerations make fast and accurate predictions of these distances a much more attractive alternative to direct measurement. For one thing, direct measurements can suffer in scalability-the amount of performance sacrificed for on-demand, large-scale proximity measurements can outweigh the performance gained by utilizing that information, defeating the purpose of collecting the measurements in the first place. Second, direct measurement can be intrusive to the network in general. A system in which many nodes independently, frequently, and simultaneously inject measurement probes into the network will incur severe overhead costs, resulting in overall network performance degradation. Third, any future value of recent and similar measurement probes as a resource is lost, causing many of the measurements to be both wasteful and redundant. For instance, there are perceivable benefits in making measurements by one node available to other nodes in the network. For these reasons and others, it is becoming more and more apparent that large-scale Internet applications could greatly benefit from some system through which network distances can be efficiently, accurately, and readily calculated, as opposed to being explicitly and expensively measured.

In this paper, we take an approach to IDP that is different from the coordinates-based approach that prevails in the research community today [1]. Specifically, in coordinatebased approaches, Internet transmission delay is embedded into a low-dimensional geometric space such that the Internet distance between two nodes can be predicted as the distance between the coordinates of those nodes in the modeled space. In our approach, we first qualitatively investigate the relationship between geographic characteristics of node-pairs and the latencies observed between them, with respect to what we shall call the node-pairs hypothesis: that groups of geographically similar node-pairs (two connected Internet hosts) will tend to exhibit similar latency characteristics.

For an example of how this simple idea might be used, refer to Fig. 1, which shows the relative placement of six nodes on a simplified geographic plane. For the node-pairs (A, B) and $(\mathrm{C}, \mathrm{D})$, the geographical distance between the nodes within either pair is the same. However, suppose that the latency between the nodes within each pair is very different. This means that the nodes in the two pairs have the same amount of geographic separation but a different amount of delay-space separation. If the latency for the third pair, $(\mathrm{E}, \mathrm{F})$, is unknown, we can predict it to be the same as the latency between $A$ and $B$ by noting the pair's geographical similarity to pair (A, B).

A simple, direct application of geographic coordinates is not a suitable option for latency estimation, because Internet paths between points are drastically different from the corresponding direct, geographic paths. However, that is not to say that all geographic properties of node links should be ignored in considering the problem of latency estimation. Based on the information learned from the evaluation of the node-pairs hypothesis, we proceed to describe and evaluate a 
mathematical model that can utilize these underlying geographic signatures to predict Internet distances between any two given Internet nodes. Finally, we discuss the reasons for why this prediction method, based on the geography of node pairs, might be preferred over other existing methods.

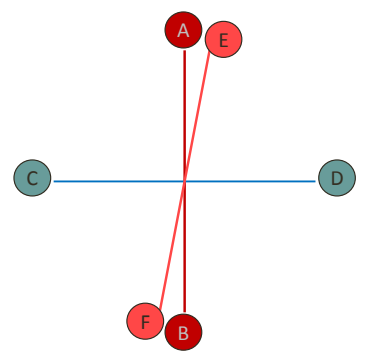

Figure 1. Three node-pairs on an Euclidean plane

\section{RELATED WORK}

The search for a resolution to the conflict between network optimization and scalability of optimization makes Internet distance prediction a very interesting and important research problem. The single most dominant flavor seen in current IDP techniques is based on the embedding of the network delayspace into one of many different types of virtualized geometric spaces to produce a coordinate system for end hosts, allowing distances to be quickly calculated based on these virtualized node coordinates. Other, simpler models, which are not coordinate-based, rely on the direct application of the triangle inequality, inferring estimated latencies of arbitrary paths based on direct measurements of a small subset of network paths. Most previous research has focused more on analyzing the correlation between geographic distance and Internet distance $[13,14,15,16,17]$, in contrast to the idea for using key geographic properties of the Internet to make network distance predictions.

The first major research effort on Internet distance prediction was based on a virtual topology method, known as IDMaps (Internet Distance Maps) [2]. In this service-oriented approach, strategically selected "landmark" nodes (called tracers), along with a distance topology of these nodes generated through direct measurement, are used to estimate the distance between any two hosts. This distance estimate is calculated as the sum of the distance between one host and its nearest tracer, the distance between the other node and its nearest tracer, and the distance of the smallest path between the two tracers as determined by tracer topology. The intention with IDMaps is not to provide fine-grain, highly accurate distance information between arbitrary nodes, but rather for IDMaps to be used as an Internet-wide distance estimation service that would allow hosts to distinguish between servers that are "very far", "very close", or in between. Thus, a distance estimate was considered successful even it was off from the actual distance by as much as a factor of two.

$\mathrm{Ng}$ and Zhang introduced the idea of a coordinate-based IDP mechanism and called their approach Global Network Positioning (GNP) [3]. Additionally, their idea involved the use of a distributed, peer-to-peer architecture, unlike the clientserver architecture used in IDMaps. The basic idea that makes a coordinate-based approach attractive is that predicting the
Internet distance between two hosts requires only that a host evaluate a distance function over the modeled geometric space, with input of its own coordinates and those of another host. This is especially suitable in a peer-to-peer setting, for dissemination of coordinates to other nodes can be achieved over the network traffic that is generated as part of the normal functioning of the system. The main algorithmic challenge in coordinate systems is two-fold: (1) how should a peer that joins the network determine the initial coordinates for itself and (2) how do all peers in the network maintain their coordinates in the face of continually changing network conditions.

This initial work in coordinate-based prediction depends on the maintenance of a set of fixed, well-trusted landmark nodes to compute coordinates for all peers. Since its appearance, a number of other coordinate systems and coordinate-based algorithms have been proposed and explored, some of which tackle the problem not with a landmark-based architecture, but a more decentralized one where all peers have equal status. These decentralized coordinate system methods have become more popular than landmarks for their potential of peer-to-peer computing and resource sharing. In the work by Costa et al. at Microsoft Research [4], any participating peer can serve as a landmark, removing the need for infrastructural support that is required, for example, by GNP. The first large-scale, peer-topeer, real world software application and content distribution service to employ a decentralized coordinate system was Vuze, a highly popular BitTorrent client. Specifically, the Vivaldi algorithm [5], which uses a mass-spring relaxation model in a Euclidean space to assign node coordinates, has been integrated into Vuze's DHT component. The purpose of Vuze's current use of Vivaldi is to evaluate the potential of the algorithm in increasing the applications DHT lookup efficiency [6].

When scrutinized from the perspective of needs of largescale systems and applications, Internet distance prediction is a very difficult problem. A level of prediction accuracy that can produce significant benefits for such applications is yet to be achieved. Several recent evaluation and comparison studies of the common and representative solutions in use today have consistently produced results showing that, regardless of what may be claimed of different accuracy metrics, the quality of these solutions is short of satisfactory from an applications perspective $[7,8,9,10,11,12,13]$. Factors like policy-based routing, congestion, circuitousness, and triangle inequality violations in Internet paths all contribute as sources of quality degradation of these algorithms.

\section{CORRELATION BETWEEN GEOGRAPHIC DISTANCE AND NETWORK DELAY}

\section{A. Data Sets Used}

We utilized three datasets to study the relationship between the geographic distance separating Internet nodes and the network delay experienced between them. One data set was collected by us using a tool called DipZoom, which provides local, programmatic access to a number of node points around the world for measuring network information (such as that provided by ping and traceroute, for example) [24]. The other two data sets we obtained from the Network Coordinates Research Group at Harvard [18]. One of these contains median latencies for 4198460 measurements across 1895 blog nodes, 
estimated using the King method [19]. The second of these data set contains median latencies for a 72-hour, all-pairs ping trace of 225 PlanetLab nodes, consisting of 50625 measurements. For each of these three data sets, the only pieces of information available for each measurement are the source and destination IP addresses and the median round-trip time (RTT). The geographic locations and distance of node pairs were not available. To obtain these, we utilized an HTTP-based IP geolocation service API [20] from which the latitude and longitude of each node in a measurement could be obtained (and thus also the distances between them). In Section 5, we comment on this process of obtaining geo-coordinates as a source of inaccuracy in prediction results.

The data collected from DipZoom is the main one used in this paper for detailed experiments. The other two data sets mentioned are discussed below briefly and used mainly for the purpose of showing that any general latency data collected over a set of globally placed nodes tends to display a similar, recurring pattern. In fact, this observation is key to the main contribution of this paper.

\section{B. Delay Symmetry Between End Hosts}

As described in Section 1, we hope to capture any unique geographic properties of predictive power that node pairs may exhibit as a unit. This goal could be compromised if the delay characteristics of inter-node communication were to display significantly asymmetrical behavior. Thus, it is first necessary to determine the extent of the risk posed by any potential delay asymmetry between nodes. One simple way to do this is to observe the absolute difference between latency values for pairs of nodes in a data set. For the DipZoom dataset, this is presented as a scatter plot of distance versus absolute difference in Fig. 2. The main observation from this plot is that the density of points is heavily contained on the zero mark for absolute difference.

It is apparent that the delay symmetry is significantly stronger for the lower range of distances, since while the number of points is very large, the mean is very close to zero and the standard deviation is very small when compared to distances in the higher ranges. The only actual troublesome range that may result in inaccuracies is the $8000-\mathrm{km}$ to $11000-\mathrm{km}$ range, where about 300 measurements display a mean absolute difference of about 15-20 milliseconds. But, fortunately, this difference level in reverse-pair delay is relatively small. This result now allows us to continue the analysis by treating the node-pair as the basic unit of data. With this understanding, the following filter was applied to all three data sets: For each node-pair appearing in the measurements, if multiple measurements exist for the same node-pair (without regard to directionality), all but the minimum-latency measurements for that node-pair were discarded.

\section{Patterns Observed in Distance-Delay Relationship}

Fig. 3, Fig. 4, and Fig. 5 present the scatter plots of RTT versus geographic distance for the DipZoom data set, the King data set, and the PlanetLab data set, respectively. For the King data, displaying about 865,000 data points, the lower-left corner of the plot seems to be polluted with unusually low latency values for distances of up to $4000 \mathrm{~km}$. At this point it is worth mentioning a key aspect of the King method of latency estimation between nodes. In the King method, the latency from node $A$ to node $B$ is estimated as the difference between the latency from node $A$ to its DNS server and the latency from node $A$ to node $B$ 's DNS server (this method is intended to address the scalability of collecting large amounts of latency data and to overcome situations where control over target nodes is not available). Perhaps this indirection in measurement can be attributed to what appears to be a strong presence of noisy data in the King data scatter plot. But we are unable to determine this for sure.

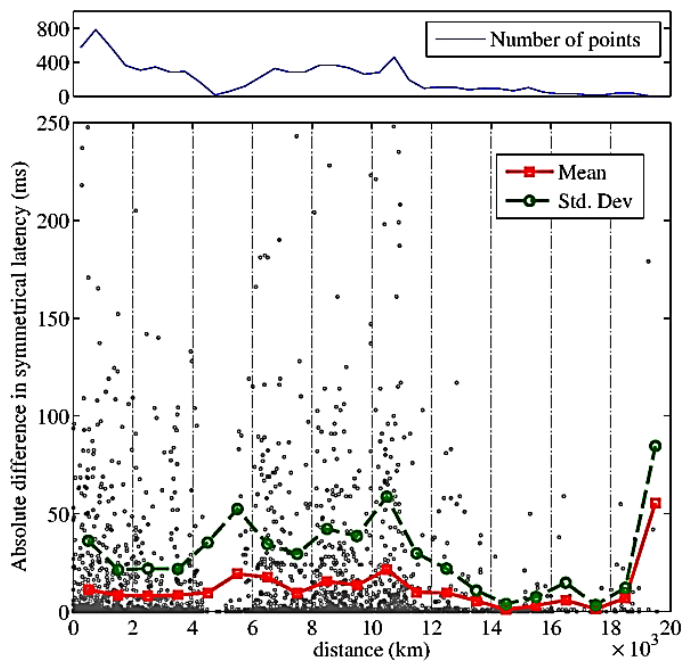

Figure 2. Scatter plot for absolute difference between bidirectional node-pair latencies as a function of inter-node distance.

In addition to the plots presented here, a number of such plots from other research studies all very strongly emphasize the same recurring pattern. For instance, two separate studies by Ziviani et al. $[15,16]$, the one in [17], and one from topology monitors at CAIDA [21], present a number of different distance-delay plots for various regions in the globe using the Test Traffic Measurements data from the RIPE network [22]. Though at first glance the three data sets in this experiment appear to generate markedly different distance-versus-delay profiles (especially the King dataset), it is important to note that all of them, and those from other studies, still share these very common features:

- There is a general slope and orientation to the collective points in each scatter plot. In other words, if one were to zoom out and view these scatter plots from a larger scale, each would display a line with slope equal to the lines of the other plots scaled similarly (but possibly with varying line thickness).

- There three or four very discernible clusters of points common across all plots. (Not so much for the King data set, but more strongly so for the DipZoom and PlanetLab data.) For example, there is the linear-form cluster in the lower-left corner of the plots, a similar linear-form cluster across the $6000-\mathrm{km}$ to $12000-\mathrm{km}$ range, and a third linear-form cluster just below those two clusters. Also, there is the "fuzzier" cluster of points in the block corresponding to distance and RTT ranges of $8000-12000 \mathrm{~km}$ and $250-350 \mathrm{~ms}$. 
- Within the "fuzzy", non-linear cluster, there is slant in the upper edge that hints to the existence of a slope equal to the slope of the other linear clusters.

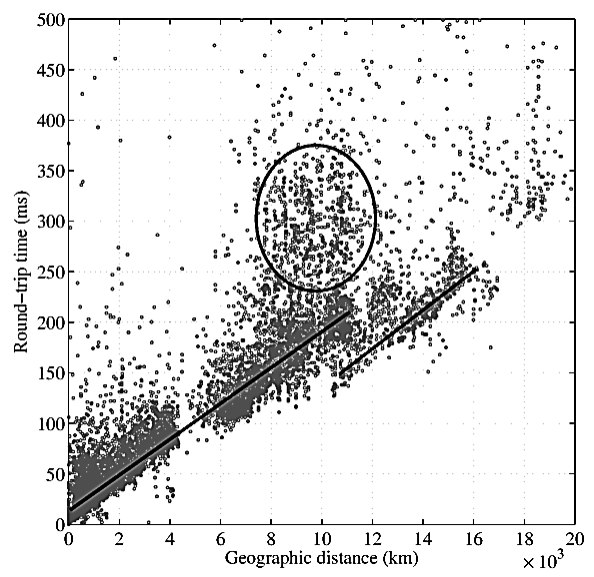

Figure 3. Geographic distance vs. network delay for the DipZoom dataset.

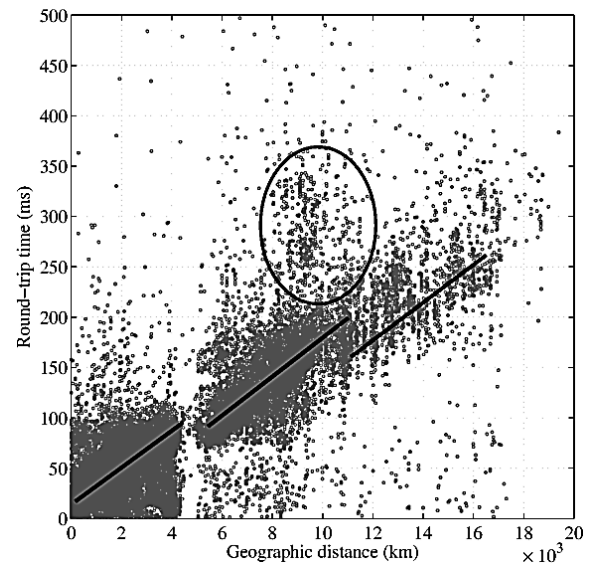

Figure 4. Geographic distance vs. network delay for the King dataset.

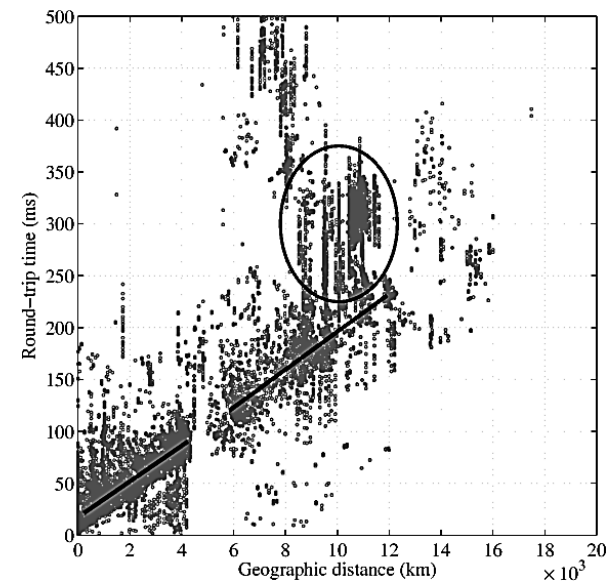

Figure 5. Geographic distance vs. network delay for the PlanetLab dataset.

There is a very common, and perhaps even universal, pattern to be exploited from these results. While a simple model based on inter-node distances alone would not be sufficient to achieve good accuracy, it is worth investigating the predictive power of a model based on these additional qualitative observations. It is these observations-along with the node-pairs hypothesis - that form the basis of the discussion in the next subsection, and also the introduction to the mathematical model in Section 4.

\section{Qualitative Geographical Characterization of Distance and Delay}

In this subsection, the goal is to characterize and make sense of what appear to be unique clusters of points in the scatter plots of distance and latency seen so far. By visualizing the characteristics of this data on an actual map of the globe, we seek to substantiate the claim that there is indeed another geographical dimension, besides distance, that directly affects the latency between Internet nodes.

In Fig. 6, the data in the scatter plot is the same as in the previous scatter plot for DipZoom (Fig. 3), but the key clusters identified before have been color coded for ease of reference and discussion. Moreover, two lines have been fitted to the linear cluster forms by manually selecting the set of points corresponding to each one. The two clusters comprising Region A were fitted together, since there is a clear connection between them. The large gap at about the 5000-km mark means that there are not many node pairs situated on the globe that are apart from each other by that particular distance. For comparison, the line corresponding to the signal speed in fiber cable (two-thirds the speed of light in a vacuum) is also shown, having slope $5 \times 10^{-3} \mathrm{~ms} / \mathrm{km}$. This is what we would see if the Internet was "perfect" (i.e., if all Internet paths were uniform and laid out in the same way as would direct geographic path between nodes on the globe). The slope of the latency data clusters, however, appears to be about $17 \times 10^{-3} \mathrm{~ms} / \mathrm{km}$. In a sense, this slope appears to be a "magic number" for observed signal rate through paths in the Internet. In order to investigate this thought further, it would help to focus on the most difficult range of distances in the scatter plot-where there correlation between distance and delay deteriorates most. As seen from the scatter plot, this happens to be the $8000-12000 \mathrm{~km}$ range. What can we expect to see by charting node-pair locations from this range on a map of the globe?

We can do this systematically in the following way: imagine a line segment, having a slope of $17 \times 10^{-3} \mathrm{~ms} / \mathrm{km}$, in the 8000-12000 km distance bracket. We move that segment up or down arbitrarily so that it lies in different latency brackets each time. For every latency bracket that we move the line segment to, a certain set of points will happen to fall around the segment. This creates different groupings of points that lie in the same distance bracket but different latency brackets. Suppose that each of those groups of points is charted in its own separate map by drawing lines between node-pairs. Then, under the node-pairs hypothesis, ideally what we should expect to see is that there is very little similarity across the different maps, if any, in the placement of connecting lines that are drawn. The more this is true, the more we can hope to come up with a method that can exploit this extra dimension of information from node-pair similarities, without relying on geographic distance alone.

In Fig. 7, a visualization tool from CAIDA, called GeoPlot, [23], is used to generate precisely the described visual representation of node-pairs as lines on a globe. To help 
determine with more confidence what points are actually connected by the lines where there is interference with other overlapping lines, all lines are color coded by distance and black dots are placed on connected node sites. Moreover, in some instances, carefully comparing the angles of similarlooking lines across different maps reveals that they are actually not connecting the same endpoints. The highlighted segments of the miniature scatter plot in the lower right of the figure indicate what node-pairs the lines in a particular map correspond to.

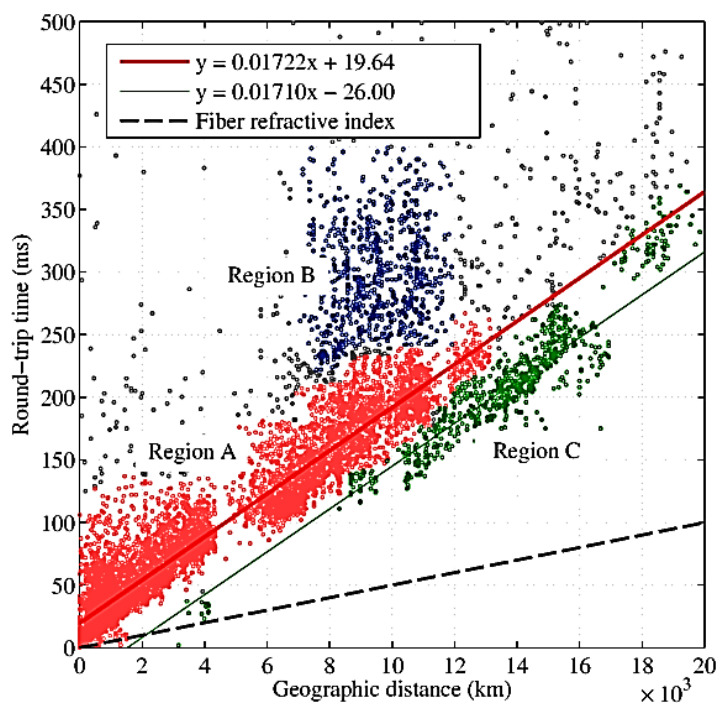

Figure 6. Color-annotated version of the DipZoom latency measurements along with lines fitted for the linear clusters.

From careful inspection of exclusivity of lines in maps (a) to (e), it is clear that almost all sets of lines are supporting examples of the type we expect to see under the node-pairs hypothesis. (This is even clearer on larger, interactive versions of the maps provided by GeoPlot, where hovering over lines causes them to be highlighted.) If any violations of the hypothesis are present in these maps, then they are very rare. One pattern in particular for which it is hard to make a decision on is the set of yellow-orange lines seen in maps (a)-(d), extending across the Pacific Ocean and between the U.S. and East Asia.

\section{A CLASSIFIER-BASED PREDICTION MODEL}

\section{A. Mathematical Formulation}

Having shown that there is potential for node-pair geography to serve as the underlying principle in a latency prediction model, we will now lay out the mathematical ideas towards constructing one.

Very naturally, the process of using node-pair signatures to predict latencies of arbitrary node pairs can be understood as a supervised learning problem. Whatever feature vector is used in devising a classifier, it must somehow capture at least the geographical information for a given node pair, and it must do so in a symmetrical way, such that there is no influence of the directionality of a node pair in the training or application of the classifier. This problem can be easily solved by applying a very simple feature transformation: if the features are simply the geo-coordinates of the two nodes in a pair, then the transformation to be applied is a primary sort on the latitude coordinates and secondary sort on the longitude coordinates. In this way, it does not make a difference if a node-pair input is presented as $(112,54),(35,67)$ or as $(35,67)$, $(112,54)$. Assuming ascending sort, for example, the transformation will cause either input to be always stored as $(35,67),(112,54)$.

What remains to be decided, however, is what the classifier should actually be made to predict. At first thought, latency itself appears to be the most obvious option. But this classifier will likely perform very poorly when compared to simple linear regression on the latency data, since the most dense distance ranges have very high correlation with latency. Also, there appears to be an inherently general slope to most of the data on distance versus latency; only for some distance ranges, the best-fit line shifts significantly below or above. This vital part of latency's relationship with distance cannot be ignored. To combine the predictive utility of node-pairs and the characteristic qualities of distance ranges to boost overall prediction accuracy for latencies, we define the output of the classifier to be the $y$-axis intercept of our general line with slope $17 \times 10^{-3} \mathrm{~ms} / \mathrm{km}$. With this approach, we can now provide a complete, abstract-level definition of a prediction model based on node-pair geography. It is summarized by the following equation:

$$
L(a, b)=\left(17 \times 10^{-3}\right) \cdot D(a, b)+C(a, b)
$$

The functions $L, D$, and $C$ are the RTT latency, the geographic distance, and the classifier output, respectively, for the geographic coordinates of two nodes given as inputs $a$ and $b$. There are many possible options for what learning function to use for $C$. For instance, one can use a regressor function instead of a classifier. For this paper, we use a simple multi-class classification strategy, which is described in detail in the next section.

\section{B. The k-nearest-node-pairs Classifier}

Our classifier is parameterized with four different types of values:

1) Set of line segments to get good coverage over the data points in the plot of distance versus delay, in a manner similar to that presented in Fig. 8. It may be possible to automate this process based on clustering techniques. It is important that the lines in the set collectively account for the entire range of distances.

2) Distance bins that divide the full range of distances into a set of smaller ranges. A number of different policies can be used for this process. For example, one approach can be to partition the distances based on all distinct subsets of overlapping line segments obtained from the previous step.

3) Number of representative points to store per line segment per distance bin. The representative points for each such line sub-segment are simply the closest data points to that segment in the particular distance bin.

4) Value of $k$. During classification, the classifier will predict the label for the most frequent of the top- $k$ nearest representative points to an input vector. 


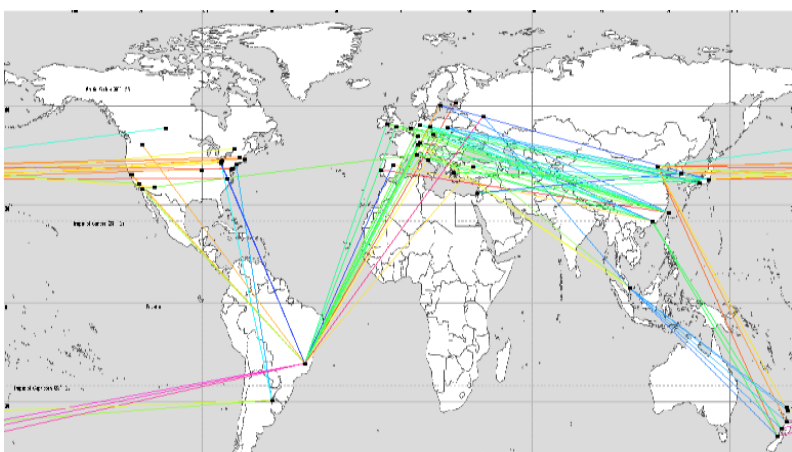

(a)

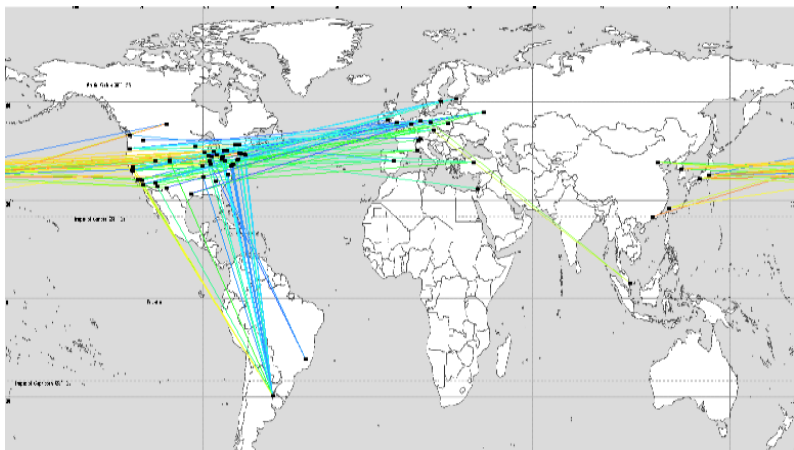

(c)

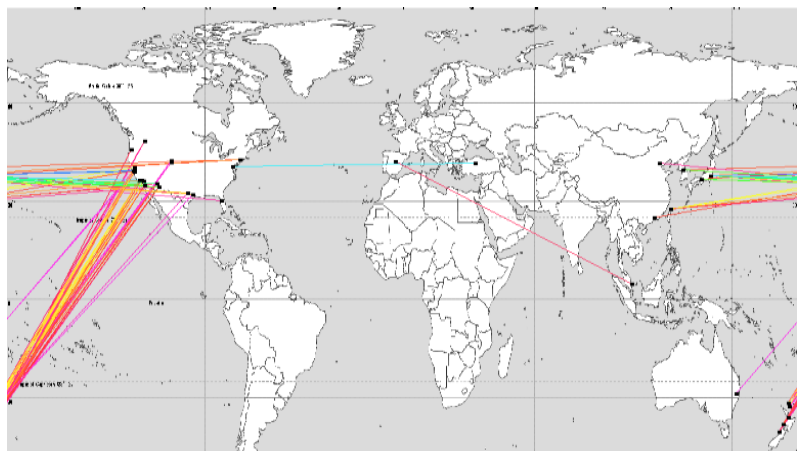

(e)

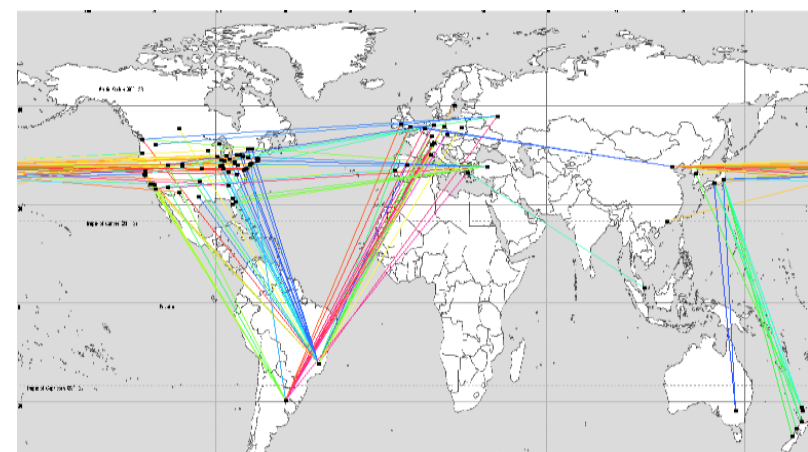

(b)

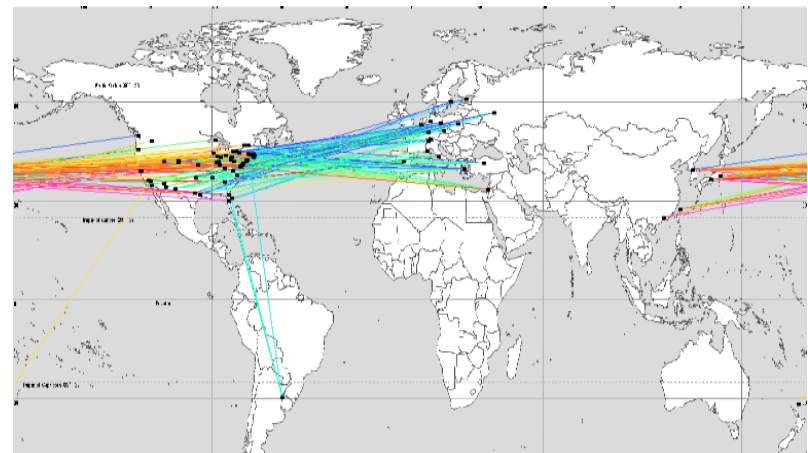

(d)

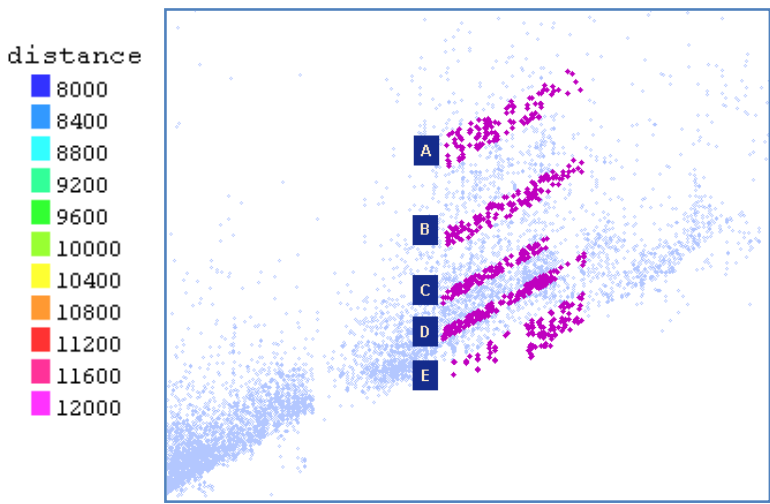

Figure 7. Geographic visualizations of node-pairs in arbitrarly selected latency brackets in the $8000 \mathrm{~km}$ to $12000 \mathrm{~km}$ range.

To make a prediction, the classifier proceeds by first identifying the subset of possible line segments that can be predicted for a particular input vector based on the distance between the pair of nodes. Next, the Euclidean distances between the input and all representative points for the subset of line segments are calculate using their geo-coordinates. Finally, a prediction is made based on the most frequently occurring line segment in the top- $k$ closest representative points to the input vector.

Our classifier was run on the DipZoom data set. The test data used for evaluating the performance of this classifier is the DipZoom data set, minus the representative points from that set obtained during training. For the classifier parameters, the set of line segments was manually constructed by visual inspection of the plot, and the outcome is presented in Fig. 8. Constantsized bins of 700-km width, 10 representative points, and $k=5$ are the values used for the other three classifier parameters.

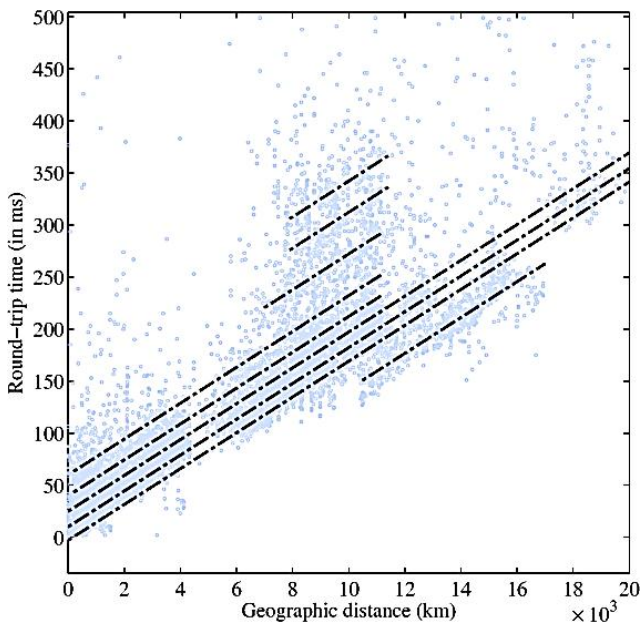

Figure 8. A sample construction for the input set of line segments to the nearest-node-pairs classifer. 


\section{Classifier Performance}

A visual form of the classifier's performance is given in Fig. 9a, and the expected result of a perfect classifier is shown on the left in Fig. 9b. The more the colors of the data points match the colors of the lines to which they are closest, the better the classifier is. There is a curiously high misclassification region for the lower distance bin up to the 4000-km mark. Perhaps a different set of training parameters would reduce the noise in this region. However, the opposite is true for the higher distance ranges. The "fuzzy", high-latency cluster of points show very good classification performance.

In Fig. 10, two different performance metrics are shown for the accuracy of this latency predictor and for the accuracy of the basic linear predictor that uses the fitted line for Region A (from Fig. 6). On the top is the relative error metric, the one that has been most commonly used in previous publications. The CDFs for both the full range of distances and the upper range (greater than $5000 \mathrm{~km}$ ) are shown. On the bottom is absolute error. According to the relative error metric, the classifier-based latency predictor performs worse than the simple linear model for the full range of possible distances. However, the relative error metric does not accurately reflect the performance for the type of problem we are trying to solve. For instance, this metric will treat a latency difference between $1 \mathrm{~ms}$ and $3 \mathrm{~ms}$ with the same degree of "poorness" as it will a latency difference of, say, $100 \mathrm{~ms}$ and $300 \mathrm{~ms}$. Moreover, this uneven representation is over emphasized in this dataset, as the highest density of points is in fact in the very lower range of latency values. Evidence for this fact is shown in the CDFs for the upper distance range.

A better metric for comparison would be the absolute value of the difference between measured and predicted latenciesthis is shown in Fig. 10b. By this standard, the classifier actually increases the overall accuracy of latency predictions.

Finally, in Fig. 11, we show the relationship between predicted and measured error for the two different latency predictors. The classifier-based approach shows a much tighter correlation when compared to the simple linear model.

\section{CONCLUSION}

Most existing solutions for IDP are based on direct, ongoing measurements of partial Internet paths, from which virtual coordinates for hosts are computed. However, this results in an accumulation of errors from incongruity of end-toend Internet paths, caused by factors such as policy-based routing by ISPs, triangle inequality violations, and network path circuitousness. These factors do not apply to the node-pair geography based approach described in this paper, since the only considerations taken into account are pairwise and local geographic properties of end-hosts. Whatever the characteristics may be of the specific path traveled by packets between two nodes, it is reasonable to expect other, geographically close nodes to display the same delay characteristics, as opposed to a very different pair of nodes that may still the same distance apart. Furthermore, the node-pair approach in this paper is able to exploit what appears to be a general measure of signal delay in the global Internet, as inferred from the very similar slopes of the clusters in distance versus latency plots.

Yet, using node-pair geographic properties does have its limitations. Perhaps the greatest source of inaccuracy in this technique comes from the method used to obtain the geocoordinates of a node from its IP address. IP geolocation is an interesting and open problem in its own right. One interesting and natural way that many Internet measurement servers and infrastructures work around this problem is by enhancing nodes with GPS capability, allowing them to determine and share their precise coordinates with other nodes in the network.

One potential idea towards improvement is this: if geocoordinates are to be determined from IP addresses as input, then one may apply the node-pair assumption directly to IP addresses and bypass the lookup for geo-coordinates altogether. Clearly, a method using this approach would require knowledge of how IP prefixes are allocated and inter-domain routing policies. Since IP addresses are the coordinates directly understood by the Internet, removing the extra indirection from geo-coordinates could result in a superior prediction model.

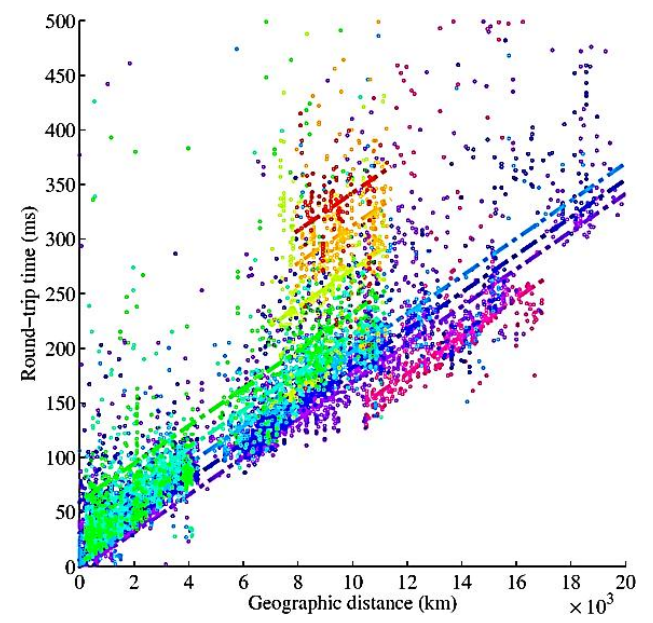

(a)

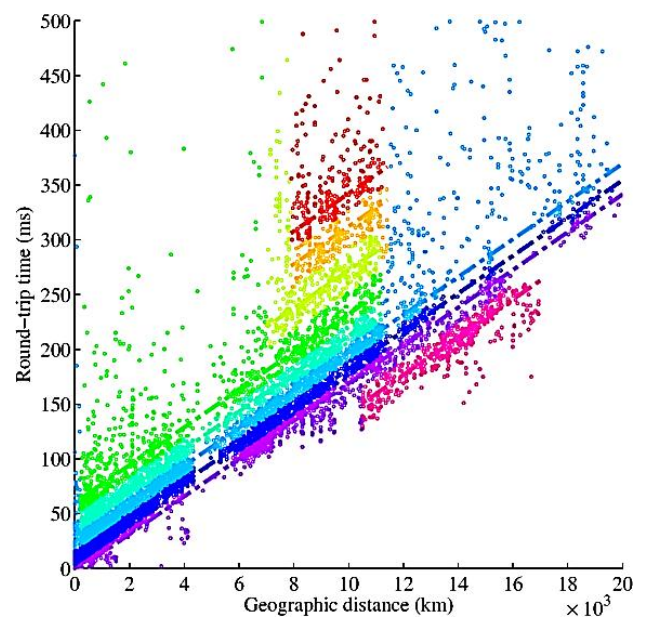

(b)

Figure 9. Classification performance of the k-nearest-node-pair classifier. Matching colors between point and line indicate higher success. 


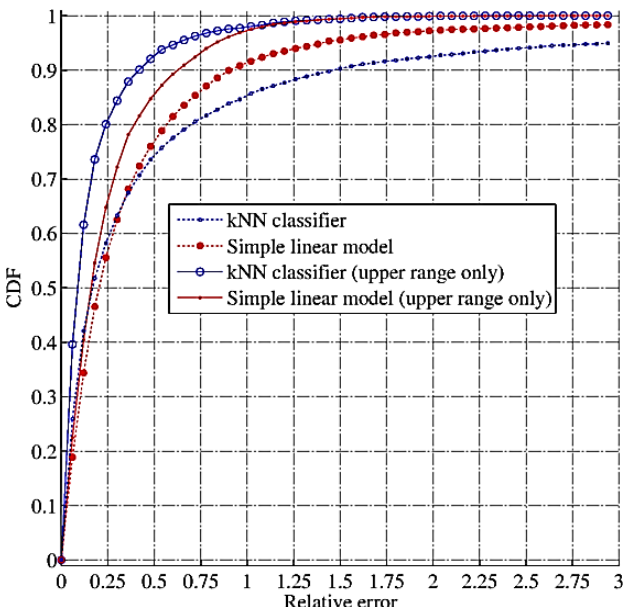

(a)

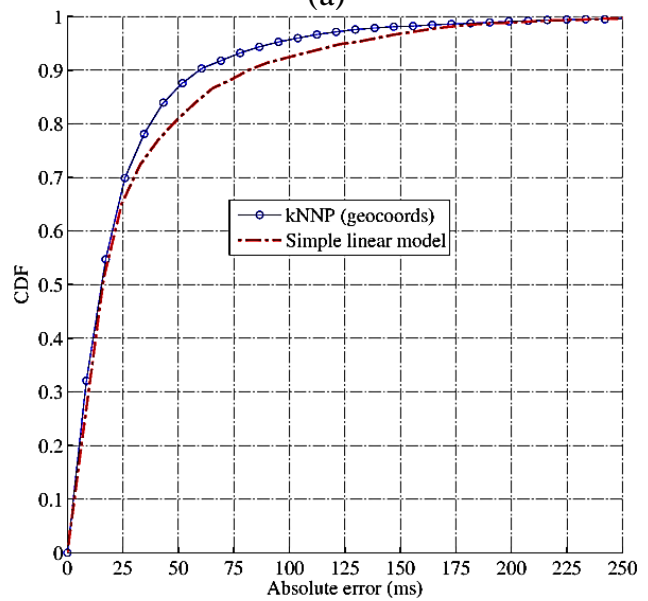

(b)

Figure 10. The relative error and absolute error CDFs for the classfier-based latency predictor and the basic linear model.
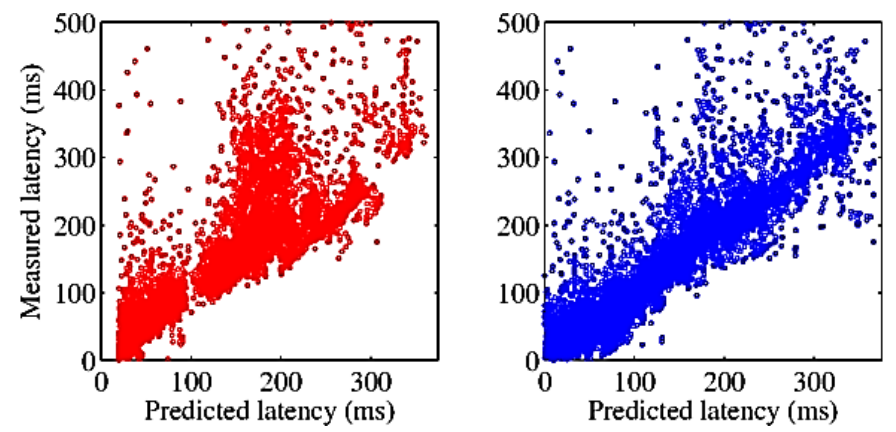

Figure 11. Measured vs. predicted latency for the basic linear model (left) and the classfier-based latency predictor (right).

While it is true that latency estimation methods based only on geographic distance cannot be generalized to achieve desirable accuracy, the results in this project show that adding another dimension based on node-pairs can help greatly. In our experiments, this other dimension happens to be based on a geographic property, but this need not be the case.

\section{REFERENCES}

[1] Z. Wen and M. Rabinovich, "Dynamic landmark triangles: A simple and efficient mechanism for inter-host latency estimation," Computer Networks, vol. 55, no. 8, June 2011.

[2] P. Francis, S. Jamin, C. Jin, Y. Jin, D. Raz, Y. Shavitt and L. Zhang, "IDMaps: a global internet host distance estimation service". IEEE/ACM Trans. Networking, Oct. 2001.

[3] T.S.E. Ng and H. Zhang, "Global network positioning: a new approach to network distance prediction," Computer Comm. Review, vol. 32, no. 1, Jan. 2002.

[4] M. Costa, M. Castro, R. Rowstron and P. Key, "PIC: Practical Internet coordinates for distance estimation," Proc. Int. Conf. on Distributed Computing Systems (ICDCS), Mar. 2004.

[5] F. Dabek, R. Cox, K. Kaashoek and R. Morris, "Vivaldi, a decentralized network coordinated system," SIGCOMM, Aug. 2004.

[6] Purpose of the Vivaldi implementation in the Vuze BitTorrent client. http://wiki.vuze.com/w/Vivaldi_View

[7] J. Ledlie, P. Pietzuch and M. I. Seltzer, "Stable and accurate network coordinates," Proc. Intl. Conf. Distributed Computing Systems (ICDCS), July 2006.

[8] E. K. Lua, T. Griffin, M. Pias, H. Zheng and J. Crowcroft, "On the accuracy of embeddings for Internet coordinate systems," Proc. Internet Measurement Conference (IMC), Oct. 2005.

[9] R. Zhang, C. Tang, Y. C. Hu, S. Fahmy and X. Lin, "Impact of the Inaccuracy of distance prediction algorithms on Internet applications," Proc. INFOCOM, Apr. 2006.

[10] R. Zhang, Y. C. Hu, X. Lin and S. Fahmy, "A hierarchical approach to Internet distance prediction", Proc. Intl. Conf. Distributed Computing Systems (ICDCS), July 2006.

[11] B. Donnet, B. Gueye and M. A. Kaafar, "A survey on network coordinates systems, design, and security," IEEE Comm. Surveys \& Tutorials, vol. 12, no. 4, Oct. 2010.

[12] C. Xing and M. Chen, "Characteristics of Internet latency and their impact on distance prediction accuracy," Proc. Comm. Networks and Services Research Conf. (CNSR), 2009.

[13] L. Subramanian, V. N. Padmanabhan and R. H. Katz, "Geographic properties of Internet routing," Proc. USENIX Annual Tech. Conf., pp. 243-259, June 2002.

[14] V. N. Padmanabhan and L. Subramanian, "An investigation of geographic mapping techniques for internet hosts," Proc. SIGCOMM, Aug. 2001.

[15] A. Ziviani, S. Fdida, J. F. de Rezende and O. C. M. B. Duarte, "Similarity models for Internet host location," Proc. IEEE Intl. Conf. on Networks (ICON) Sept. 2003.

[16] A. Ziviani, S. Fdida, J. F. de Rezende and O. C. M. B. Duarte, "Toward a measurement-based geographic location service," Proc. Passive \& Active Measurement Workshop (PAM), Apr. 2004.

[17] B. Huffaker, M. Fomenkov, D. J. Plummer, D. Moore and K. Claffy, "Distance metrics in the Internet," Proc. Intl. Telecomm. Symp. (ITS), 2002.

[18] Network Coordinates Research Group at Harvard, http://www.eecs.harvard.edu/ syrah/nc/

[19] K. P. Gummadi, S. Saroiu, and S.D. Gribble, "King: Estimating latency between arbitrary internet end hosts," Proc. SIGCOMM Internet Measurement Workshop, 2002.

[20] IPInfoDB, an IP Geolocation service. http://ipinfodb.com/

[21] CAIDA topology monitor: Otp-ro, Evolva Telecom, Bucharest, RO. http://www.caida.org/projects/ark/statistics/otp-ro.html

[22] RIPE Test Traffic Measurements. http://www.ripe.net/ttm/

[23] Geographic visualization tool from CAIDA. http://www.caida.org/tools/visualization/geoplot/

[24] M. Rabinovich, S. Triukose, Z. Wen, and L. Wang, "DipZoom: The Internet measurement marketplace," Proc. IEEE Global Internet Symp., 2006. 